\title{
Evaluation of a cricothyroidotomy simulator for teaching extreme surgical airway management with and without ultrasound
}

\author{
S. Ninan, J. Macallan, B. Birk, E. Walton, J. Willers, D. Uncles
}

Department of Anaesthesia and Intensive Care, Worthing Hospital, Lyndhurst Road, Worthing, West Sussex, United Kingdom

\section{Background}

NAP 4 showed that anaesthetists' surgical airway(SA) skills were poor and this needs to be addressed by increased training via simulation. It became apparent that there were no models available in conditions where SA is most urgently needed, conditions like burns, ${ }^{(1)}$ anaphylaxis and angioneurotic oedema, where ultrasound(US) use has been suggested.(2)

A cost effective high fidelity model was developed for extreme SA so we decided to evaluate it to see if it could fill the gap in currently available training. (3)

\section{Method}

We evaluated a cricothyroidotomy simulator modified to replicate soft tissue neck swelling by adding an ADAMgel (Aqueous Dietary fibre Antifreeze Mix gel) layer, as a training tool using an established questionnaire for validating SA models. 12 anaesthetists with simple SA training ( 3 consultants, 3 associate specialists, 3 senior and 3 junior trainees) performed surgical cricothyroidotomies without and with US guidance following a sonoanatomy demonstration in normal neck anatomy.

Figure 1 below shows cricothyroidotomy placement using the ADAMgel simulator and figure 2 demonstrates sonoanatomy as seen on the airway model compared with normal human landmarks.
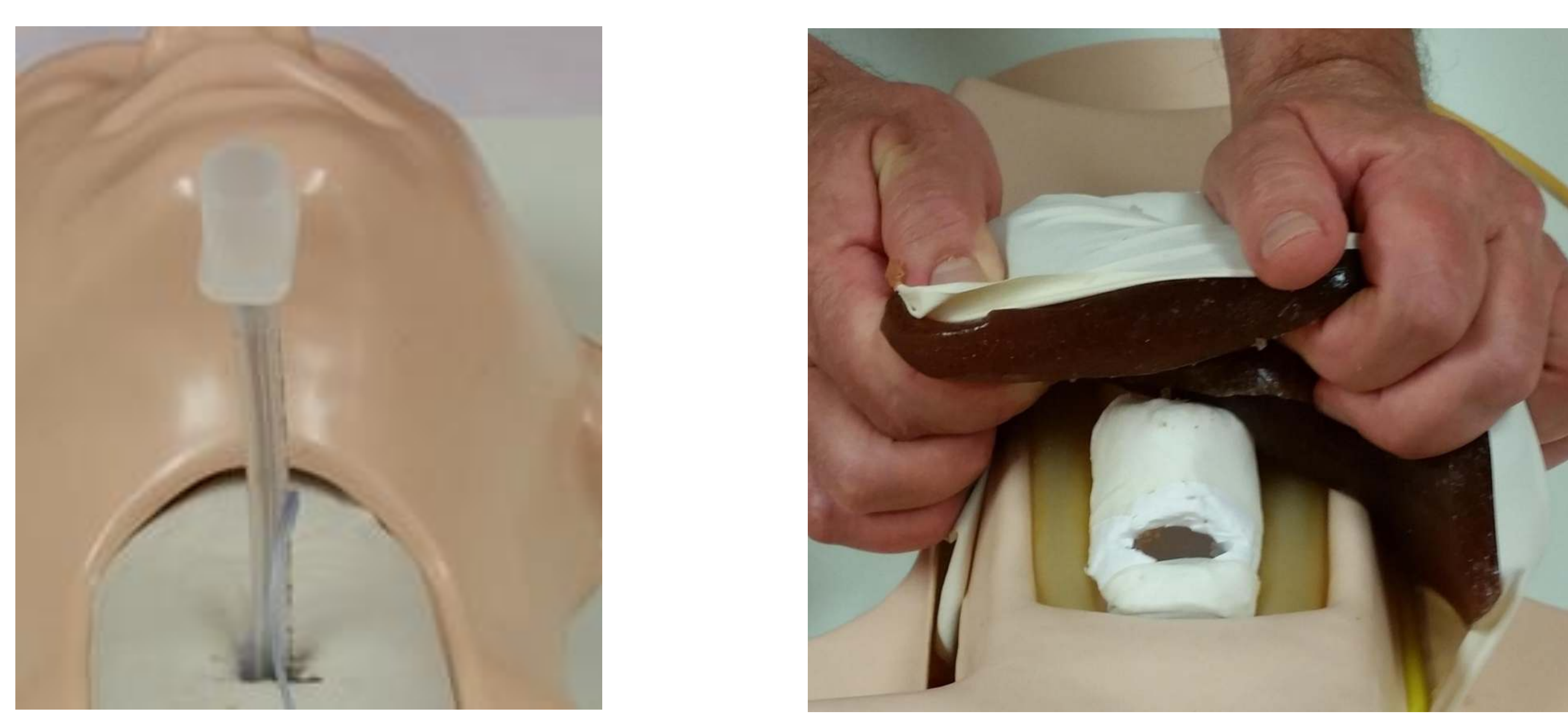

Figure 1. Cricothyroidotomy performed with ultrasound guidance



Figure 2. Collage of sonographs showing anatomical structures in the neck pericricothyroid in two age differentiated live subjects (top - old and bottom - young, employing additional ADAMgel to enhance soft-tissue swelling) and the airway model (middle).

\section{Results}

12 participants enrolled in this study and all completed questionnaires for both airway scenarios. The results were universally positive.(Fig. 3) All participants felt this model was particularly useful in training more junior clinicians before they 'practised' on patients. 1 consultant and a senior trainee had difficulty performing the procedure without US but all were able to do it with US guidance.



Figure 3. Feedback from evaluation of both airway simulation models

\section{Discussion}

This is a cost effective model that is reusable, recyclable, and the first of its kind. It enables high fidelity simulation training of extreme surgical airways with ultrasound. Most participants reported increased confidence with equipment, performing the procedure on patients, and felt it resembled real life.

\section{Conclusion}

This study demonstrates the utility of this extreme surgical airway model with and without US. It would lead to an increase in confidence and competence despite previous lack of exposure to these difficult and potentially lifethreatening situations.

\section{References}

1. T. Howes ,C. Lobo,F. Kelly, T. Cook. Rescuing the obese or burned airway: are conventional training manikins adequate? A simulation study. Br J Anaesth. Jan 2015;114(1):136-42.

2. K Curtis, Mahern, M Dawson, M Mallin. Ultrasound-guided, bougie-assisted cricothyroidotomy: A description of a Novel Technique in Cadaveric Models. Academic Emergency Medicine, 2012;19:876-879.

3. Willers J, Roberts A, Staniforth M, et al. Ultrasound assisted extreme surgical airway simulation. BMJ STEL. 2015;1:Suppl 2 A28. 